\title{
Les anthelminthiques : utilisation en médecine vétérinaire
}

\author{
par J. BUSSIERAS $(*)$
}

\begin{abstract}
RESUME
L'auteur présente une liste des principaux anthelminthiques modernes, suivie de commentaires sur les particularités de leur utilisation vétérinaire, et sur les problèmes liés à leur emploi en Afrique.
\end{abstract}

La thérapeutique anthelminthique, en médecine vétérinaire comme en médecine humaine, s'est profondément transformée depuis une vingtaine d'années. Les produits anciens, souvent d'origine végétale (extrait de fougère mâle, essence de chénopode, etc.) ont progressivement été remplacés par des substances de synthèse; actuellement, de nouveaux anthelminthiques sont systématiquement recherchés et testés par les grands laboratoires pharmaceutiques, si bien que les progrès dans ce domaine sont rapides.

II serait long et fastidieux de faire une étude complète sur les anthelminthiques maintenant abandonnés ou en voie de l'être; c'est pourquoi nous nous limiterons à une récapitulation des substances les plus utilisées actuellement, avant d'étudier les particularités de leur emploi en médecine vétérinaire, et les problèmes propres à leur utilisation en Afrique tropicale.

\section{LES PRINCIPAUX ANTHELMINTHIQUES MODERNES}

\subsection{Anthelminthiques actifs sur les Nématodes}

Les Nématodes les plus importants chez les animaux domestiques en Afrique sont avant tout les parasites du tube digestif : strongles (y compris ankylostomes), ascarides, trichures, anguillules, spirures.

La phénothiazine, qui fut très utilisée surtout chez les Ruminants, est maintenant moins employée; en effet, certaines espèces d'helminthes étaient peu sensibles à son action, d'autres devenaient chimio-résistantes; en outre, sa toxicité n'était pas négligeable et elle donnait parfois des accidents de photo-sensibilisation.

Par contre, les sels de pipérazine, aux propriétés connues depuis longtemps également, conservent tout leur intérêt dans l'indication précise du traitement des ascaridoses.

Il est curieux de remarquer que la quasi totalité des produits d'utilisation récente, à l'exception des organo-phosphorés, sont des composés hétérocycliques, dont la molécule contient azote et soufre (comme la phénothiazine elle-même d'ailleurs).

(*) Professeur de l'Ecole Inter-Etats des Sciences et Médecine Vétérinaires, Dakar, Sénégal. 
Chaque substance sera désignée par sa dénomination commune, suivie du nom des auteurs ayant les premiers publié une étude sur la question; enfin seront mentionnées quelques appellations commerciales. Les posologies indiquées sont généralement celles préconisées par les fabricants; leur adaptation à l'Afrique tropicale sera envisagée plus loin.

\subsubsection{Dérivés de l'imidazole}

- Le thiabendazole: connu depuis 1961 (6); Thibenzole, Nemapan.

Modes d'utilisation. - Par voie buccale, à la posologie de 50 à $110 \mathrm{mg} / \mathrm{kg}$, chez les Ruminants, Equins et Porcins; administré de force (suspension aqueuse, comprimés) ou en mélange dans la nourriture (granulés).

Avantages. - Très bonne efficacité sur la plupart des strongles digestifs adultes, sur certaines de leurs formes larvaires (Haemonchus sp.), et sur les anguillules (Strongyloides sp.). Très bonne tolérance, coefficient thérapeutique particulièrement élevé.

Inconvénients. - Action limitée sur les ascarides, très faible ou nulle sur les strongles respiratoires et sur les trichures. Non utilisable par voie parentérale.

- Le tétramisole: (signalé par THIENPONT et Collab., 38); Nemicide, Anthelvet, Vermium, Vadephen, Nilverm, Ripercol, Citarin, Concurat.

Modes d'utilisation. - Voie buccale, $10-15 \mathrm{mg} / \mathrm{kg}$ (comprimés ou solution), voies parentérales (sous-cutanée, intramusculaire ou intrapéritonéale), $5-7,5 \mathrm{mg} / \mathrm{kg}$.

Avantages. - Possibilité d'injections parentérales; efficacité sur strongles digestifs, strongles respiratoires, ascarides, anguillules...

Inconvénient. - Marge de sécurité relativement faible. L'utilisation du lévamisole (Ripercol-L, Solaskil), isomère gauche du tétramisole, et seule forme active, permettra de réduire posologie et toxicité, en conservant toute l'efficacité.

- Le parbendazole (1), Padovex, Helmatac, Niverzol, est employé chez les Ruminants et les Porcins, per os, à la posologie de $30 \mathrm{mg} / \mathrm{kg}$.

- Le cambendazole, signalé par HOFF et Collab. (24) a une très bonne efficacité sur les strongles digestifs des Ruminants et des Porcins, à la dose de $20 \mathrm{mg} / \mathrm{kg}$ par voie buccale.

- Le mébendazole $(7,18)$, Telmin, est utilisé chez les Equidés, per os, à la dose de 5 à $13 \mathrm{mg} / \mathrm{kg}$.

Ces trois derniers produits présentent certains avantages et inconvénients du thiabendazole, mais avec une efficacité semble-t-il supérieure, ce qui permet d'abaisser la posologie et d'obtenir une action sur les stades larvaires; en outre le cambendazole se révèle extrêmement polyvalent, agissant aussi sur les strongles respiratoires (33) et même sur les petites douves du foie (35) et sur les Cestodes Anoplocéphalidés (10).

Enfin le parbendazole est relativement efficace sur les trichures du porc (37).

\subsubsection{Dérivés de la pyrimidine}

- Le pyrantel (2) : Exhelm, Banminth.

Modes d'utilisation. - Surtout employé sous forme de tartrate, par voie buccale, dans les diverses espèces de mammifères domestiques à la posologie de 10 à $25 \mathrm{mg} / \mathrm{kg}$ (poudre mêlée aux aliments, ou solution). Dans le cas des carnivores, ont aussi été expérimentés le pamoate et le chlorhydrate.

Avantages. - Très bonne activité sur les strongles digestifs, les ascarides, les anguillules. Très bonne tolérance. 


\section{Inconvénients. - Cf. Thiabendazole.}

- Le morantel (9); Exhelm II, Banminth II, Bovhelm, Ovithelm, dérivé méthylé du précédent, a une efficacité supérieure qui permet de limiter sa posologie à $6-10 \mathrm{mg} / \mathrm{kg}$.

Tout récemment enfin (HOWES, 1972), le «CP-14.445 », autre dérivé du pyrantel, a été annoncé comme particulièrement actif contre les trichures chez le chien et le porc.

\subsubsection{Composés organo-phosphorés}

Dans le groupe des composés organo-phosphorés, à propriétés anticholinestérasiques, ont été mis au point un certain nombre d'anthelminthiques relativement bien tolérés par les animaux supérieurs :

- Le dichlorvos, Atgard, Equigard, Canogard, Task, Tenac.

Mode d'utilisation. - Employé sous forme de granulés plastiques qui libèrent lentement le principe actif tout au long du tube digestif; dose de 30 à $40 \mathrm{mg} / \mathrm{kg}$, administrée par voie buccale, principalement aux équins, aux porcins, et aux chiens.

Avantages. - Polyvalent, très bonne activité sur les strongles digestifs (y compris ankylostomes et osophagostomes), ascarides, oxyures, trichures; actif même sur des larves de diptères (Gasterophilus sp. des Equidés); moins actif sur les anguillules.

- Le naphthalophos (Maretin, Rametin), l'haloxon (Galloxon), le coumaphos (Baymix), le trichlorfon (Neguvon), sont également utilisables.

Toutes ces substances doivent être employées en respectant une posologie assez stricte, sous peine d'accidents liés au blocage des cholinestérases.

\subsection{Anthelminthiques actifs sur les Trématodes}

En Afrique tropicale, sont surtout à considérer les médicaments actifs sur les grandes douves du foie, Fasciola gigantica, et, en second lieu, ceux détruisant les paramphistomes. Petites douves du foie et bilharzies justifient rarement un traitement chez les animaux domestiques.

Les produits actifs sont actuellement extrêmement nombreux et de structure chimique souvent voisine, la plupart d'entre eux ayant fait suite à l'hexachlorophène (13) maintenant moins utilisé à cause d'une relative toxicité.

Ces substances sont généralement dérivées de la salicylanilide, ou parfois du diphényle, du diphényl sulfoxyde, du nitrophénol.

\subsubsection{Substances actives sur les formes adultes de Fasciola}

- Le bithionol, ou bitin (39), D $2 \mathrm{~N}$, Actamer. Voie buccale, $30 \mathrm{mg} / \mathrm{kg}$.

- Le bithionol sulfoxyde, ou bitin-S (30), Disto-5, Neo-Distol. Voie buccale, $40 \mathrm{mg} / \mathrm{kg}$.

- Le niclofolan (27), Bilevon-R, Bilevon-M. Voie buccale, $3 \mathrm{mg} / \mathrm{kg}$, ou sous-cutanée, $1 \mathrm{mg} / \mathrm{kg}$.

- Les mélanges de bromsalan et tribromsalan (28), Hilomid, Mitenyl, Diaphene. Voie buccale, $20-30 \mathrm{mg} / \mathrm{kg}$.

- L'oxyclozanide (41), Zanil. Voie buccale, $15 \mathrm{mg} / \mathrm{kg}$.

- Le nitroxynil (11), Dovenix, Trodax. Voie sous-cutanée ou intramusculaire, $10 \mathrm{mg} / \mathrm{kg}$.

- Le clioxanide (8), Tremerad. Voie buccale, $15 \mathrm{mg} / \mathrm{kg}$.

- Le bromophénophos (40), Acedist. Voie buccale, $15-20 \mathrm{mg} / \mathrm{kg}$.

- Le rafoxanide (29), Ranide, Flukanide. Voie buccale, $7,5 \mathrm{mg} / \mathrm{kg}$.

- Le brotianide (32), Dirian. Voie buccale, $5-7 \mathrm{mg} / \mathrm{kg}$. 


\subsubsection{Substances actives sur les formes jeunes de Fasciola}

Les formes jeunes de la grande douve, souvent fort pathogènes (risques de fasciolose aiguë), sont très résistantes à la plupart des thérapeutiques qu'il serait nécessaire d'employer à des doses subtoxiques.

Cependant, à des posologies usuelles ou légèrement augmentées, on obtient (4) pour Fasciola hepatica, dans des conditions expérimentales, la destruction:

- des douves de 6 semaines et plus, avec le nitroxynil,

- des douves de 4 semaines et plus, avec le rafoxanide.

\subsubsection{Substances actives sur les paramphistomes}

Tous les douvicides modernes mériteraient d'être expérimentés dans le traitement des paramphistomoses des Ruminants.

Ont déjà montré leur efficacité, même sur les formes immatures (seules pathogènes) :

- le niclofolan, $6 \mathrm{mg} / \mathrm{kg}$,

- le bithionol, $30 \mathrm{mg} / \mathrm{kg}$,

- la niclosamide (cf. ci-dessous), $50-100 \mathrm{mg} / \mathrm{kg}$.

\subsection{Anthelminthiques actifs sur les Cestodes}

Comme en médecine humaine, chez les animaux les anciens ténifuges ont en grande partie été supplantés par un dérivé de la salicylanilide: la niclosamide, Yomesan, Lintex, Mansonil, Tredemine, utilisée dans toutes les espèces domestiques per os à une posologie de 50 à $125 \mathrm{mg} / \mathrm{kg}$.

La tolérance est bonne, mais chez le chien l'efficacité est insuffisante sur Echinococcus granulosus, contre lequel il est toujours nécessaire d'utiliser, comme autrefois, le bromhydrate d'arécoline.

Sont également utilisables contre les Cestodes des animaux domestiques: la bunamidine (Scolaban), le résorantel (Terenol) et un di-isothiocyanate (Cetovex).

Remarquons enfin que certains fasciolicides (Bithionol notamment) sont également actifs sur divers Cestodes parasites des ruminants.

\section{LES PARTICULARITES DE L'UTILISATION VETERINAIRE DES ANTHELMINTHIQUES}

Le traitement anthelminthique des animaux domestiques pose un certain nombre de problèmes qui le distinguent nettement de celui destiné aux humains. Ces problèmes sont liés aux anthelminthiques eux-mêmes, aux espèces parasites et aux animaux à traiter.

\subsection{Problèmes liés aux anthelminthiques}

\subsubsection{La production des anthelminthiques vétérinaires}

Elle est caractérisée par l'importance des débouchés commerciaux; en effet, dans de nombreux pays développés, les helminthoses animales ont toujours une grande importance alors que les humains ne sont que peu ou pas parasités. Ces conditions influencent la commercialisation et même la recherche de nouveaux anthelminthiques. Contrairement à de nombreux autres domaines, où la recherche thérapeutique a pour objectif la santé humaine, les applications vétérinaires n'apparaissant qu'ensuite, on peut dire qu'en matière d'anthelminthiques l'objectif est souvent en premier lieu la santé animale. 
C'est ainsi que diverses substances (phénothiazine, thiabendazole, tétramisole, niclosamide, etc.) ont d'abord été connues comme médicaments vétérinaires, avant d'être employées chez l'homme.

\subsubsection{Nécessité d'une rentabilité des traitements}

Le besoin d'une rentabilité caractérise presque toute la médecine vétérinaire, à l'exception de celle des petits animaux "de luxe" (carnivores domestiques, oiseaux de volière, etc.).

En particulier, tout traitement anthelminthique à but curatif doit :

- être d'un coût très inférieur à la valeur de l'animal traité;

- être d'une efficacité telle que l'éleveur puisse en apprécier les résultats.

Autrement dit, la diminution des taux d'infestations helminthiques ne devrait pas être considérée comme le but du traitement, mais seulement comme un moyen, le but véritable étant l'amélioration des productions (baisse de mortalité, gain de poids, augmentation de la fécondité, amélioration de la production laitière). Cette notion, qui paraît évidente, est trop souvent oubliée, lorsqu'on apprécie l'efficacité d'un anthelminthique uniquement sur la diminution du nombre des vers ou de la production de leurs œufs.

\subsubsection{Toxicité et métabolisme des anthelminthiques}

En thérapeutique vétérinaire, il ne faut pas se soucier seulement de la toxicité d'un produit nouveau pour l'animal traité; plus important encore est le problème de la toxicité pour l'homme du produit lui-même ou de ses métabolites, de leur persistance dans les viandes, de leur passage dans le lait ou dans les œufs.

C'est pourquoi, pour chaque anthelminthique nouveau devraient être précisées les durées pendant lesquelles sont proscrits, après traitement, l'abattage des animaux de boucherie ou la collecte du lait ou des aufs. Des observations récentes (23) montrent ainsi que niclofolan, oxyclozanide et nitroxynil chez la vache s'éliminent pendant une à deux semaines dans le lait à des concentrations telles qu'il convient de prohiber leur utilisation chez les sujets en lactation.

\subsection{Problèmes liés aux espèces parasites}

\subsubsection{Multiplicité des espèces}

On est frappé en médecine vétérinaire par l'extrême multiplicité des formes parasites qui peuvent être présentes chez l'animal.

C'est ainsi que, dans le vaste ensemble des strongles digestifs, essentiellement représentés chez l'homme par deux espèces d'ankylostomes, il est possible de trouver chez un même animal des dizaines d'espèces distinctes; généralement, ces diverses espèces n'offrent pas la même sensibilité aux anthelminthiques.

\subsubsection{Importance des stades immatures}

Des helminthoses très graves (fasciolose aiguë, paramphistomose aiguë, ๙sophagostomose) sont provoquées par des larves ou par des adultes immatures; or ces formes sont généralement beaucoup moins sensibles aux anthelminthiques que les adultes mûrs, obligeant ainsi à employer des doses élevées, parfois subtoxiques, des médicaments. Par exception, on observe, dans les paramphistomoses des Ruminants, une bonne activité de la niclosamide sur les stades immatures, pathogènes, alors que le même produit est inactif sur les vers adultes non pathogènes (25); de même, une substance nouvelle, le diamphénétide (12), ou Coriban, se révèle paradoxalement plus efficace sur les formes jeunes que sur les adultes de la grande douve du foie.

Par ailleurs, dans de nombreuses helminthoses, et notamment les strongyloses, on constate que le développement endogène des larves de surinfestation se trouve bloqué par la présence d'adultes préexistants. Or l'utilisation des anthelminthiques usuels, qui détruisent principalement 
les vers adultes, a pour résultat de permettre à ces larves de terminer leur développement et donc de reconstituer rapidement la population initiale. Ce fait oblige à traiter au moins une deuxième fois, après un délai de quelques semaines.

C'est pourquoi, comme le remarque GIBSON (14), l'emploi des anthelminthiques récents, généralement plus coûteux mais suffisamment actifs sur la plupart des formes larvaires de strongles, peut constituer une véritable économie, en permettant d'administrer un traitement unique.

\subsection{Problèmes liés aux animaux-hôtes}

\subsubsection{Grande diversité des espèces à traiter}

Les espèces animales susceptibles d'intéresser le vétérinaire sont extrêmement variées, depuis les petits oiseaux de volière jusqu'aux grands mammifères. Ce fait conduit à choisir les anthelminthiques, non seulement en fonction des espèces parasitaires en cause, mais aussi des espèces hôtes; en effet des anthelminthiques sont mal tolérés par certains animaux (tétrachlorure de carbone chez les bovins, tétrachloréthylène chez les équidés, etc.).

\subsubsection{Modes d'administration des anthelminthiques}

Le mode d'administration sera choisi en fonction de l'espèce animale (d'une contention plus ou moins facile), du nombre d'individus (problème des traitements collectifs) et aussi bien entendu de la nature du produit à administrer.

- La voie buccale: simple et pratique lorsque le médicament peut être administré en mélange dans la nourriture, elle oblige dans les autres cas (administration au pistolet-doseur, etc.) à une bonne contention des animaux; de plus elle expose à certaines erreurs de posologie; enfin, chez les Ruminants, le produit administré risque de se diluer considérablement dans la panse.

- Les voies sous-cutanée et intramusculaire ne sont utilisables qu'avec une minorité d'anthelminthiques modernes (tétramisole, nitroxynil); elles permettent l'administration de doses plus précises, mais obligent à des précautions d'asepsie, et sont parfois à l'origine de réactions locales.

\subsubsection{Moment du traitement}

La vie en troupeaux oblige à choisir pour le traitement anthelminthique des animaux une date moyenne, qui peut être précisée à l'avance en fonction des conditions épidémiologiques habituelles dans la région considérée (traitement "stratégique »), ou au contraire être fixée chaque fois que les conditions climatiques font craindre une recrudescence des infestations (traitement " tactique »).

\subsubsection{Cas particuliers des élevages industriels}

Le développement des élevages industriels pour certaines espèces animales (porc, poulet) a pour conséquences :

- la raréfaction ou même la disparition de certaines helminthoses, principalement celles dues à des parasites à cycle indirect; en effet, les locaux aménagés pour ces élevages constituent des milieux généralement défavorables au développement des invertébrés hôtes intermédiaires;

- une augmentation du risque d'autres helminthoses, principalement celles dues à des parasites à cycle direct; leur transmission est en effet facilitée par l'état de surpeuplement dans lequel sont placés les animaux.

Or il est indispensable d'éviter tout retard de croissance, toute baisse de production, qui seraient catastrophiques pour la rentabilité de ces installations.

On est alors conduit à essayer de remplacer les traitements curatifs, administrés trop tardivement, par des méthodes de chimio-prévention; celles-ci, qui consistent à utiliser l'anthelmin- 
thique à très faibles doses quotidiennes dans la ration, sont rendues possibles par l'emploi d'aliments composés préparés industriellement; c'est ainsi que l'hygromycine B est depuis longtemps utilisable dans la prophylaxie des ascaridoses.

\section{PARTICULARITES DE LUUTILISATION AFRICAINE DES ANTHELMINTHIQUES VETERINAIRES}

Les conditions très particulières de l'élevage en Afrique tropicale obligent à résoudre un certain nombre de problèmes, en vue de l'adaptation des méthodes étudiées précédemment.

\subsection{Problèmes liés aux parasites}

\subsubsection{Particularités de la faune helminthique d'Afrique tropicale}

Certains genres d'helminthes sont représentés dans les pays tropicaux par une espèce légèrement différente de celle connue dans la zone tempérée. Par exemple, les grandes douves du foie appartiennent à l'espèce Fasciola gigantica, et non pas Fasciola hepatica; cela ne modifie que peu la thérapeutique, la sensibilité des deux espèces aux douvicides paraissant généralement comparable. C'est ainsi que les doses efficaces sur les douves adultes restent identiques à celles préconisées dans les pays tempérés pour le bithionol (19), le nitroxynil (22), l'oxyclozanide (20), le bromophénophos (21), le rafoxanide (5). De même, la bonne activité sur les formes immatures de $F$. gigantica est confirmée pour le nitroxynil $(22 ; 3)$ et le rafoxanide (5).

D'autres genres sont presque limités aux pays tropicaux (Schistosoma par exemple).

Inversement enfin, des helminthes de grande importance vétérinaire sont pratiquement absents d'Afrique tropicale : strongles respiratoires, certains strongles digestifs (genres Ostertagia, Nematodirus, etc.).

\subsubsection{Fréquence des associations parasitaires}

Le bétail africain est souvent dans un état de grande déficience, due à la fois à la sousalimentation et aux infestations parasitaires; cette déficience, à son tour, augmente la réceptivité à d'autres parasitoses, si bien que la plupart des animaux sont atteints d'un grave polyparasitisme.

Cela conduit, sur le plan thérapeutique, à rechercher de préférence :

- soit des anthelminthiques suffisamment polyvalents, telles les substances actives à la fois sur cestodes intestinaux, douves hépatiques et même paramphistomes (bithionol par exemple); à signaler aussi les propriétés du nitroxynil et du rafoxanide, efficaces à la fois sur les grandes douves hépatiques et sur certains strongles digestifs (Haemonchus, etc.); dans le cas du rafoxanide, l'activité s'étend même aux estres de l'appareil respiratoire du mouton (31); rappelons enfin que le cambendazole s'annonce comme extrêmement prometteur;

- soit des associations médicamenteuses, destinées le plus souvent à atteindre à la fois douves hépatiques et nématodes du tube digestif; ces associations sont parfois prévues par les fabricants eux-mêmes (par exemple rafoxanide + thiabendazole : Ranizole; oxyclozanide + tétramisole : Nilzan).

\subsection{Problèmes liés aux animanx}

\subsubsection{Faible valeur commerciale de la plupart des animaux}

On observe un décalage très sensible entre les prix africains, très bas et relativement stables, du bétail et de la viande, et les prix européens, élevés et soumis à l'inflation, des anthelmin- 
thiques modernes. Aussi convient-il de calculer le prix de revient de chaque traitement, et de vérifier qu'il n'est pas disproportionné avec la valeur de l'animal traité.

\subsubsection{Plus grande sensibilité des animaux africains aux médicaments}

Par suite de différences d'espèces (zébus au lieu de taurins) ou de races, on peut observer en Afrique tropicale des accidents toxiques avec des doses d'anthelminthiques en principe inoffensives. Ces accidents sont possibles chez des animaux en bon état et, a fortiori, chez les sujets très déficients que l'on est souvent amené à traiter.

C'est surtout avec des douvicides que l'on observe de telles intolérances; autrement dit, avec ces substances, les doses toxiques sont souvent beaucoup plus basses que chez les animaux des pays tempérés, alors que nous avons vu précédemment (3.1.1.) que les doses efficaces étaient généralement comparables.

Par exemple sont signalés en Afrique avec le niclofolan chez le zébu des troubles graves dès la dose de $5 \mathrm{mg} / \mathrm{kg}$, et des accidents mortels à $10 \mathrm{mg} / \mathrm{kg}$ (17); de même le bithionol est-il parfois mal toléré par le mouton (15).

Par contre, les marges de sécurité restent très larges chez les bovins africains avec le rafoxanide, bien toléré à la dose de $45 \mathrm{mg} / \mathrm{kg}$ (36) et avec le nitroxynil (pas de troubles à $30 \mathrm{mg} / \mathrm{kg}$ ) (22).

Parmi les substances actives contre les nématodes, la plupart sont utilisables chez les Ruminants africains sans problème de toxicité.

Par contre, chez les Equidés, une expérimentation portant sur 153 ânes (16) a permis de préciser l'intérêt du thiabendazole et de l'haloxon, alors que dichlorvos, pyrantel et tétramisole sont considérés comme trop toxiques.

\subsubsection{Indocilité des animaux}

Les bovins de la plupart des races africaines (zébus et taurins) sont des sujets difficiles, peu abordables. Ce caractère inné est aggravé par le fait que les animaux sont peu habitués à être approchés, manipulés.

Il est donc souvent nécessaire, pour des traitements anthelminthiques collectifs, d'utiliser des parcs avec couloirs de vaccination; la vermifugation peut d'ailleurs être alors associée à d'autres opérations prophylactiques. Dans ces conditions, les produits injectables par voie souscutanée ou intramusculaire seront souvent d'une administration plus facile.

$\mathrm{Au}$ contraire, toutes les fois que le produit devra être utilisé par les éleveurs eux-mêmes, il sera préférable d'éviter les préparations injectables et de recommander l'utilisation de la voie buccale.

\subsection{Problèmes liés aux anthelminthiques}

En dehors des critères d'efficacité, d'innocuité et de prix de revient, déjà envisagés, les anthelmintiques doivent présenter un certain nombre de qualités pour être employés sous les climats tropicaux :

\subsubsection{Stabilité physico-chimique}

Même dans les conditions les plus défavorables de chaleur et d'humidité, les produits utilisés doivent conserver pendant des délais suffisants :

- leur efficacité,

- leur aspect physique (comprimés, poudres, etc.). 


\subsubsection{Facilité de transport et de stockage} brantes.

La préférence sera donnée à des emballages peu fragiles, à des présentations peu encom-

\subsubsection{Facilité d'utilisation}

Lorsque les anthelminthiques doivent être administrés par les éleveurs eux-mêmes, on évitera les formes liquides, souvent difficiles à faire ingérer si l'on ne dispose pas du matériel approprié (pistolets-doseurs); en outre, l'eau risque de faire défaut pour préparer extemporanément des suspensions aqueuses; enfin celles-ci peuvent être à l'origine d'erreurs de posologie, par manque d'homogénéité.

Dans ces conditions, bien préférables sont les présentations solides (comprimés, tablettes); l'idée de proposer un même produit sous deux types de comprimés de couleur différente, correspondant à deux dosages du principe actif, est à encourager.

Dans tous les cas, on évitera les substances dont l'emploi nécessite le calcul d'une posologie trop rigoureuse.

Enfin, on recherchera des médicaments ne nécessitant aucune diète préalablement à leur administration.

\subsection{Problèmes liés aux modes d'élevage}

Le nomadisme des troupeaux s'observe traditionnellement dans la plupart des pays d'Afrique occidentale.

Il se justifie d'une part, par la recherche de la nourriture (descente vers le sud pendant la saison sèche), d'autre part, en sens inverse, par la fuite devant les vecteurs de maladies, et principalement les glossines inoculatrices de trypanosomes, dès le début de la saison des pluies.

Ce nomadisme a pour conséquences :

- de limiter considérablement les réinfestations, à la façon d'une rotation des pâturages, puisque les animaux passent régulièrement sur des zones nouvelles, où la plupart des formes infestantes des helminthes ont disparu;

- mais aussi de compliquer, voire de rendre impossible, la répétition des traitements.

La sédentarisation de certains troupeaux, permise notamment par l'aménagement de points d'eau permanents, modifie progressivement ces données, en augmentant beaucoup les risques de réinfestations.

\subsection{Problèmes liés aux conditions climatiques}

Les zones d'élevage d'Afrique occidentale connaissent chaque année une alternance très marquée entre une saison des pluies, de quelques mois, et une saison sèche beaucoup plus longue.

La saison des pluies est la seule pendant laquelle les animaux trouvent une nourriture abondante, et acquièrent donc un bon état de résistance aux maladies; mais en même temps cette période est la plus favorable au développement exogène des helminthes, donc à la transmission des helminthoses.

Il s'ensuit que ces parasitoses se transmettent principalement en saison des pluies, mais que les troubles les plus graves dus aux vers s'observent en période de disette, donc en saison sèche. Ce fait est confirmé par les observations de SEIFERT (34) en Australie, qui montrent que les helminthoses gastro-intestinales des bovins n'ont pas d'effet perceptible sur la croissance des jeunes tant que les pâturages sont satisfaisants; au contraire, lorsque ces derniers s'appauvrissent excessivement, et que la croissance est ralentie chez tous les animaux, un traitement antiparasitaire permet une reprise de celle-ci. 
On peut alors se demander quel est le moment le plus favorable au traitement anthelminthique des troupeaux.

On remarquera que par suite de la grande régularité annuelle des climats tropicaux, il n'est pas possible de distinguer ici traitement stratégique et traitement tactique, qui se superposent.

Si l'on se base sur un rythme moyen de deux traitements anthelminthiques systématiques chaque année, on peut admettre que les périodes les plus favorables sont:

- la fin de la saison sèche, en vue de tarir les sources de parasites qui risquent de jouer leur rôle néfaste pendant la période pluvieuse;

- la fin de la saison des pluies, pour essayer de débarrasser les animaux des infestations qu'ils auront malgré tout contractées. parasites.

Mais ces données doivent évidemment être adaptées à chaque région et à chaque type de

Enfin, il convient d'extraire de ce programme général le cas des jeunes animaux et notamment des veaux, qui doivent être traités contre ascaridose et anguillulose non pas en fonction de la saison mais en fonction de leur âge; le moment optimal pour un traitement unique se situe vers l'âge de 3 à 4 semaines.

\section{SUMMARY}

\section{Anthelmintics : their veterinary use}

The author records the principal modern anthelmintics; he then discusses the pecularities of their veterinary and African use.

\section{RESUMEN}

\section{Los antihelminticos : Utilización en medicina veterinaria}

El autor da una lista de Ios principales antihelmínticos modernos. Pués, discute las particularidades de su utilización veterinaria y de su uso en Africa.

\section{BIBLIOGRAPHIE}

1. ACTOR (P.) et Collab. New broad spectrum anthelminthic, methyl 5 (6) - butyl - 2 - benzimidazole carbamate. Nature, London, 1967, 215 (5098): 321-322.

2. AUSTIN (W.C.) et Collab. Pyrantel tartrate, a new anthelmintic effective against infections of domestic animals. Nature, London, 1966, 212 (5067): 1273-1274.

3. BERGER (J.). A comparison of the activity of some fasciolicides against immature Fasciola gigantica in experimentally infected calves. Bull. epizoot. Dis. Afr., 1971, 19 (1) : 37-44.

4. BORAY (J.C.). Fortschritte in der Bekämpfung der Fasciolose. Schweizer Arch. Tierheil., 1971, 113 (7): 361-386.

5. BOUCHET (A.), DAYNES (P.) et BIRGI (E.). Etude de l'action anthelminthique d'un dérivé halogéné de la Salicylanilide vis-à-vis de Fasciola gigantica. Rev. Elev, Méd. vét. Pays trop., 1971, 24 (4): $543-549$.

6. BROWN (H. D.) et Collab. Antiparasitic drugs. IV 2 - (4' - Thiazolyl) - Benzimidazole, a new anthelmintic. J. Am. Chem. Soc., 1961, 83: 1764.

7. CALlEAR (J. F. F.) et NEAVE (R. M.S.). The clinical use of the anthelmintic Mebendazole. Brit. vet. J., 1971, 127 (9): XLI-XIIII.

8. CAMPBELL (A.) et Collab. A new compound effective against acute fascioliasis in sheep. Experientia, 1967,23 (12) ; 992-993.

9. CORNWELL (R.L.) et JONES (R.M.). Controlled laboratory trials in sheep with the anthelmintic morantel. Brit. vet. J., 1970, 126 (3) : 142-148.

10. CVETKOVIC (L.), LEPOJEV (O.) et ARMACKI (S.). Action du cambendazole sur les helminthes du tractus digestif du mouton, avec mention particulière des Anoplocephalidae (en serbocroate). Vet. Glasn., $1972,26(2): 91-95$.

11. DAVIS (M.), LUCAS (J. M.S.), ROSENBAUM (J.) et WRIGHT (D. E.). 4 - Cyano - 2 - iodo - 6 nitrophenol: a new fasciolicide. Nature, 1966, 211 (5051): 882-883.

12. DICKERSON (G.), HAREENIST (M.) et KINGSBURY (P. A.). A chemotherapeutic agent for all stages of liver fluke disease in sheep. Brit. vet. J., 1971, 127: XL. 
13. DORSMAN (W.). A new treatment of cattle against liver flukes (Fasciola hepatica). Rap. 16 ${ }^{e}$ Congrès int. vét., Madrid, 1959, 2 : 609-610.

14. GIBSON (T.E.). Advances in veterinary anthelmintic medication. Advances in Parasifology, 1969,7 : 349-373.

15. GRABER (M.). Région de recherches vétérinaires et zootechniques d'Afrique centrale, rapport annuel 1967, t. III. Farcha, Fort-Lamy, I.E.M.V.T., 1967.

16. GRABER (M.). Etude, dans certaines conditions africaines, de l'action antiparasitaire du Thiabendazole et de divers anthelminthiques actuels: IV. Helminthoses et gastérophiloses digestives de l'âne, Rev. Elev. Méd. vét. Pays trop., 1972, 25 (1): 53-71.

17. GRABER (M.), EUZEBY (J.) et BIRGI (E.). Essais de traitement en Afrique Tropicale de la distomatose hépatobiliaire du zébu à Fasciola gigantica. Valeur du Bilevon R Bayer. Rev. Elev. Méd. vét. Pays trop., 1970, 23 (3): 337-345.

18. GUILHON (J.), COURADEAU (G.), BARNABE (R.). Activité anthelminthique d'un nouveau dérivé de l'imidazole sur les nématodes parasites du tube digestıf du cheval. Bull. Acad. vét., 1971, 44 (7): 311-315.

19. GUILHON (J.) et GRABER (M.). Action du Bithionol sur les Amphistomes et sur Fasciola gigantica. Bull. Acad. vét., 1962, 35 (7) : 275-278.

20. GUILHON (J.) et GRABER (M.). Action d'un dérivé chloré de la salicylanilide, en milieu tropical, sur les Trématodes parasites des Bovins. Rev. Elev. Méd. vét. Pays trop., 1971, 24 (3): 365-371.

21. GUILHON (J.), GRABER (M.) et BIRGI (E.). Etude du pouvoir anthelminthique du Bromophénophos à l'égard de divers endoparasites du mouton et du zébu de la République du Tchad. Rev. Elev. Méd. vét. Pays trop., 1970, 23 (2): 199-206.

22. GUILHON (J.), GRABER (M.) et BIRGI (E.). Action du Nitroxynil sur divers parasites du zébu en Afrique centrale. Rev. Elev. Méd. vét. Pays trop., 1970, 23 (3): 347-359.

23. HEESCHEN (W.), TOLLE (A.) et BLUTHGEN (A.). Fasciolizid in Milch. Archiv. Lebensmittelhyg., $1972,23(1): 1-7$.

24. HOFF (D. R.) et Collab. A new broad spectrum anthelmintic : 2 - (4 - thiazolyl) 5 - isopropoxycarbonylamino-benzimidazole. Experientia, 1970, 26: 550-551.

25. HORAK (I. G.). The anthelmintic efficacy of Lintex and freon against Paramphistomum spp. in sheep and cattle. J. S. Afr. vet. med. Ass., 1964, 35 (2): 161-166.

26. HOWES (H. L.), Jr., Trans - 1, 4, 5, 6 - tetrahydro-2- (3-hydroxystyryl) -1-methyl pyrimidine (CP-14.445), a new antiwhipworm agent. Proc. Soc. exp. Biol. Med., 1972, 139 (2): 394-398.

27. KUTTLER (K. L.), MATTHEWS (N.J.) et MARBLE (D. M.). Comparative therapeutic efficacy of Carbon Tetrachloride, Hexachloroethane, and ME 3625 in Fasciola hepatica infections in sheep. Am. J. vet. Res., 1963, 24 (98) : 52-58.

28. LIENERT (E.). Diaphene wirkt beim Fasciola hepatica Befall des Rindes. Tierärztl. Umsch, 1963,18 (2) : 85.88.

29. MROZIK (H.) et Collab. A new agent for the treatment of liver fluke infection (fascioliasis). Experientia, $1969,25(8): 883$.

30. ODA (A.). Treatment of liver-fluke infection in cattle with A-K T 501 (Bitin-S). Nakakambara Livestock Hygiene Service Center, 1962.

31. RONCALLI (R. A.), BARBOSA (A.) et FERNANDEZ (J.F.). The efficacy of rafoxanide against the larval stages of Oestrus ovis in sheep. Vet. Rec., 1971, 88 (11) : 289-290.

32. ROSEBY (F. B.) et BORAY (J.C.). The anthelmintic efficiency against Fasciola hepatica and the toxicity of Bay 4059 in sheep. Aust. vet. J., 1970, 46 (7): 308-310.

33. RUBIN (R.). Efficacy of Cambendazole against lungworm (Dictyocaulus viviparus) of cattle. Am.J.vet. Res., 1971, 33 (2): 425-426.

34. SEIFERT (G. W.). Ecto-and endoparasitic effects on the growth rates of zebu crossbred and British cattle in the field. Aust. J. Agric. Res., 1971, 22 (5) : 839-850.

35. SIBALIC (S.), LEPOJEV (O.) et MIKLIJAN (S.). Action du cambendazole sur Dicrocoelium dendriticum chez les moutons infestés naturellement (en serbo-croate). Vet. Glasn., 1971, 25 (11): 835-839.

36. SNIJDERS (A.J.), HORAK (I. G.) el LOUW (J.P.). Trials with rafoxanide. 2. Efficacy against Fasciola gigantica in cattle. J.S. Afr. vet. med. As.s., 1971, 42 (3) : 253-257.

37. TAFFS (L. F.). Anthelmintic activity of parbendazole in swine naturally infected with lungworms and gastro-intestinal nematodes. Res. vef. Sci, 1970, 11, 515-522.

38. THIENPONT (D.) et Collab. Tetramisole (R. 8299), a new potent broad spectrum anthelmintic. Nature, London, 1966, 209 : 1084-1086.

39. UENO (H.), WATANABE (S.) et FUJITA (J.). Etudes sur des anthelminthiques contre la grande douve du foie. I. Action de quatre diphényl méthanes et de trois diphénylsulfures halogénés (en japonais). J. Jap. vet. med. Ass., 1959, 12: 297-301.

40. VAN DER MEER (S.) et POUWELS (H.). 4, 4', 6, 6' tetrabromo-2, 2' - biphenyldiol mono (dihydrogen phosphate), a new agent for combating distomatosis. J. med. pharm. Chem., 1969, 12 (3): 534-535.

41. WALLEY (J.K.). Oxyclozanide (3, 3', 5, 5', 6-pentachloro-2, 2' - dihydroxy benzanilide - «Zanil ») in the treatment of the liver fluke Fasciola hepatica in sheep and cattle. Vet. Rec., 1966, 78 (8): $267-276$. 